\title{
OBSERVATIONS OF MAGNETIC FIELDS IN QUIESCENT PROMINENCES
}

\author{
EINAR TANDBERG-HANSSEN \\ High Altitude Observatory, National Center for Atmospheric Research,* Boulder, Colo., U.S.A.
}

\begin{abstract}
The longitudinal component of the magnetic field, $B_{\|}$, has been recorded in about 135 quiescent prominences observed at Climax during the period 1968-1969. The measurements were obtained with the magnetograph which records the Zeeman effect on hydrogen, helium and metal lines. The following lines were used, $\mathrm{H} \alpha ; \mathrm{He}_{1}, \mathrm{D}_{3}, \mathrm{He}_{1}, 4471 \AA ; \mathrm{Na} \mathrm{I}, \mathrm{D}_{1}$ and $\mathrm{D}_{2}$, and the observed magnetic field component in these prominences was independent of the line. The overall mean value of the field $B_{\|}$for all the prominences was 7.3G. As a rule, the magnetic field enters the prominence on one side and exits on the other, but in traversing the prominence material, the field tends to run along the long axis of the prominence.
\end{abstract}

\section{Introduction}

It is of considerable interest to study prominence magnetic fields using the Zeeman effect on lines of different elements. In this study we have used the $\mathrm{H} \alpha$ hydrogen line, neutral helium lines $\left(D_{3}\right.$ at $5876 \AA$ and the $4471 \AA$ line $)$ as well as several metal lines $\left(\mathrm{Na} \mathrm{I}, \mathrm{D}_{1}\right.$ and $\mathrm{D}_{2}$ at $5889 \AA$ and $5896 \AA, \mathrm{Mg} \mathrm{I}, b_{1}$ at $5184 \AA$ ). Since June 1968 helium and metal line observations of prominence magnetic fields have been secured on a nearly routine basis. In addition, many hundred $\mathrm{H} \alpha$ observations also are available for comparison.

The original version of the High Altitude Observatory solar magnetograph at Climax, Colorado has been described by Lee et al. (1965). The instrument measures the longitudinal component of the magnetic field through the Zeeman effect on the $\mathrm{H} \alpha$ line, by recording the difference between the oppositely circularly polarized $\sigma$-components of the line. The instrument is servoed to let the entrance slits to the magnetograph seek out the proper intensities in the two wings, regardless of line asymmetries. In 1967 the instrument was modified and put under computer control, allowing the measurements to be recorded on magnetic tape. The tapes are processed with the CDC 6600 computer of the National Center for Atmospheric Research. Also, a new entrance slit assembly was constructed that allows narrow metal lines to be used (Lee et al., 1969).

The accuracy of the measurements depends both on the nature of the object under observation and on the spectral line used. If the prominence is very active, the servoing of the Doppler movements leads to a noisy signal. Typically, the accuracy is about $\pm 0.5 \mathrm{G}$ for $\mathrm{H} \alpha, 1 \mathrm{G}$ for $\mathrm{He} \mathrm{I}, \mathrm{D}_{3}$, and $2 \mathrm{G}$ or more for metal lines in quiescent prominences. All measurements discussed here pertain to emission lines in prominences seen above the solar limb.

The Zeeman effect displaces a $\sigma$-component of the line used from its normal

* The National Center for Atmospheric Research is sponsored by the National Science Foundation. 
position $\lambda_{0}$, by an amount $\Delta \lambda_{B}$, which is related to the magnetic field $B$ by the expression

$$
\Delta \lambda_{B}=\frac{e B \lambda_{0}^{2}}{4 \pi m_{e} c^{2}} g,
$$

where $g$ is the Landé $g$-factor, and $e$ and $m_{e}$ are the absolute charge and mass of an electron. For the fairly complicated atomic transitions in question we compute mean $g$-factors, $\bar{g}$, by weighting the individual $g$-factors by the relative intensities of the $\sigma$-components of the lines (see for instance White (1934) p.220). The magnetograph's data acquisition routine treats all data as if recorded with the $H \alpha$ line, and a correction factor must be applied to the output data for any other line.

One of the difficulties in calibrating the magnetic observations is to properly assess the influence of different processes on the line profile. Considerable effort has been employed to study the Hanle effect (Hanle, 1924) and its influence on measurements on prominence magnetic fields (Hyder, 1968; Stenflo, 1969; Lamb, 1970; and House, $1970 \mathrm{a}, \mathrm{b})$. For the lines affected, the mutual perturbations of the atomic levels alter the observed polarization. This, in turn, can be interpreted as caused by a magnetic field. The magnitude of the effect differs widely for different lines. In the following we have neglected the effect.

\section{Observations, $\mathrm{H} \alpha$}

The data analyzed pertain to the 1968-69 calendar years. Altogether, about $1100 \mathrm{H} \alpha$ observations in slightly more than 400 prominences have been studied.

Of these prominences about 135 were considered quiescent and they are treated here in more detail. Figure 1 shows a histogram of the distribution of $\bar{B}_{\|}$for this class of prominences. We find that the overall mean value is $7.3 \mathrm{G}$, and $52 \%$ of the prominences have mean values satisfying the relation

$$
3 \mathrm{G} \leqslant \bar{B}_{\|} \leqslant 8 \mathrm{G} \text {. }
$$

Rust (1966) found $\left\langle\bar{B}_{\|}\right\rangle \approx 5 \mathrm{G}$ for data from 1965 , and Harvey (1969) found $\left\langle\bar{B}_{\|}\right\rangle=$ 6.6G. His data were obtained mainly in 1967. The differences between the mean values quoted $(\approx 5 \mathrm{G}, 6.6 \mathrm{G}$ and $7.3 \mathrm{G})$ may not be significant, and may be due to selection effects. On the other hand, Harvey pointed to the possibility that the general level of prominence-supporting fields could have been greater in 1967 than closer to sunspot minimum in 1965, thereby explaining the difference between his and Rust's results. The present study supports this point of view, and it will be of interest to continue this type of observation to see if the level will decline again as we progress past the 1969 solar maximum.

When we observe the magnetic field in prominences with only one spectral line, say $\mathrm{H} \alpha$, a series of measurements of the field from many different parts of the object is often possible. We find then as a general rule that even though the field may change some in strength across the prominence, the sense of direction $(-B$ or $+B)$ is the same. Those relatively rare instances where we see both polarities may be explained in 


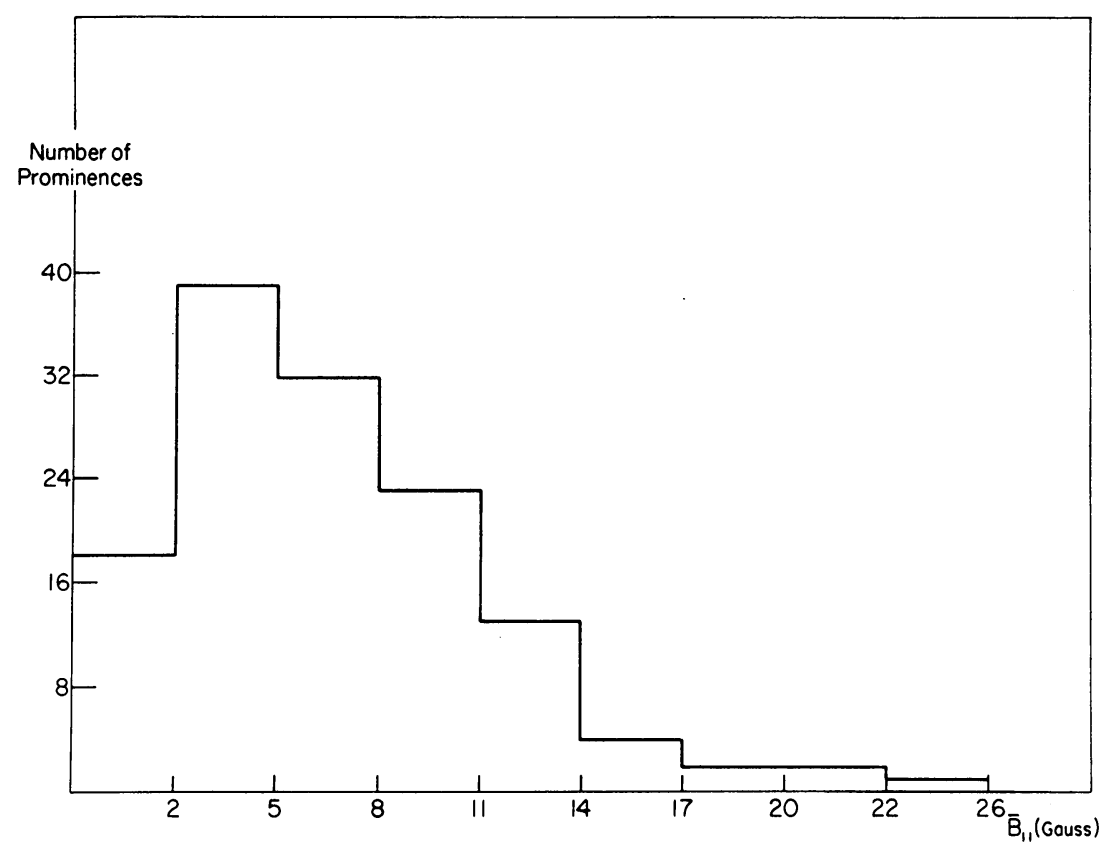

Fig. 1. Histogram giving the distribution of mean longitudinal magnetic field $\bar{B}_{\|}$for 135 quiescent prominences observed in 1968-69.

terms of geometry (the prominence sheet is bent) or localized activity in the prominence.

Apart from these situations, we observe the magnetic field either as it enters one side of the prominence ( $B$ negative), or as it emerges ( $B$ positive). Figure 2 shows some typical examples.

Rust (1966) and Harvey (1969) found that the observed longitudinal field tends to increase with height in the prominence. The present study confirms this general tendency, see Figure 3, but there are many prominences where this increase is masked by internal noise in the data.

\section{Observations Using Several Different Spectral Lines}

If we want information about the magnetic field using several different spectral lines, often there is time only to make the measurements on a few selected spots in the prominence. Table I summarizes the pertinent data for a number of quiescent prominences whose magnetic field has been determined by observing two or more of the hydrogen, helium or metal lines mentioned in Section 1.

The most striking result of a perusal of Table $I$ is the impression that the magnetic field is the same whether observed with hydrogen, helium, or metal lines, i.e.

$$
B_{\|}(\mathrm{H} \alpha) \approx B_{\|}\left(\mathrm{D}_{3}\right) \approx B_{\|}\left(\mathrm{D}_{1}\right) .
$$




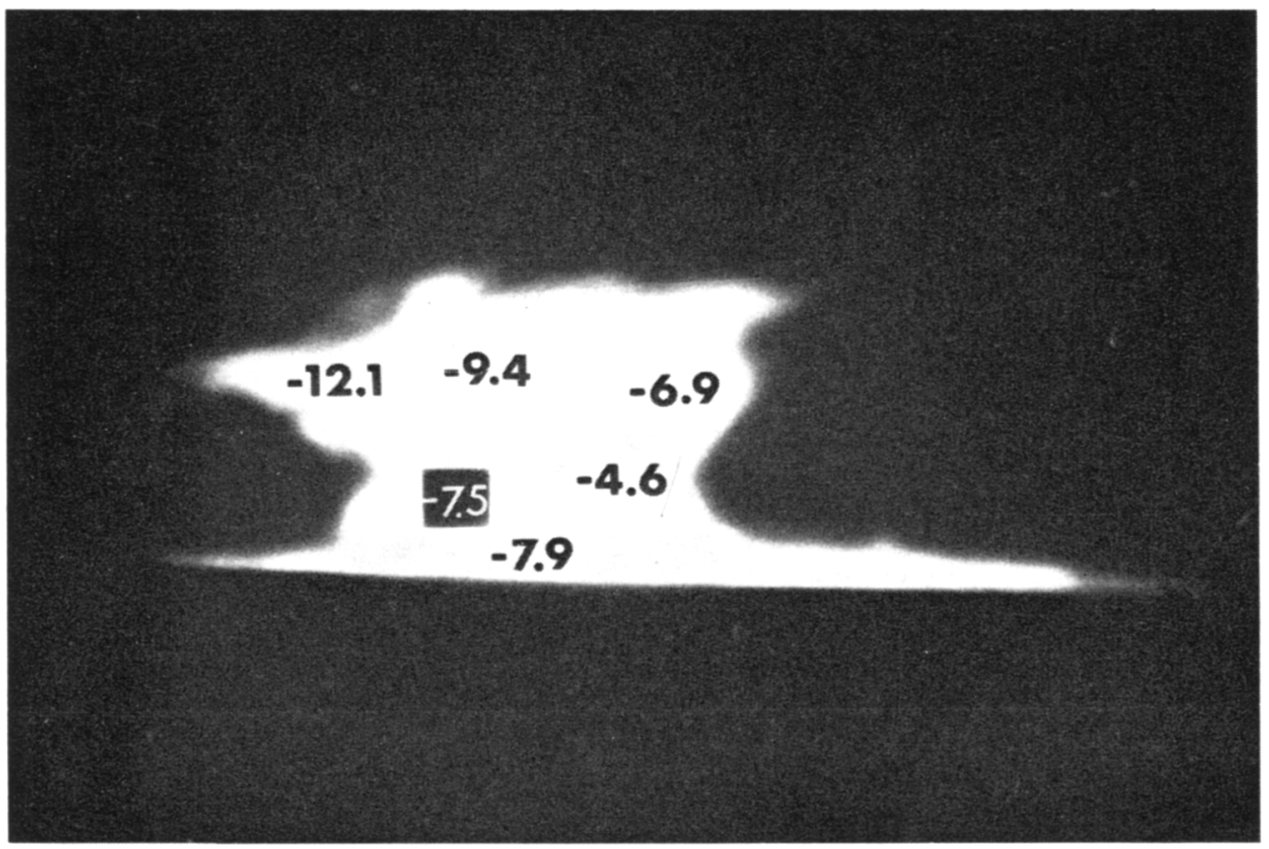

\section{March 3, 1968}

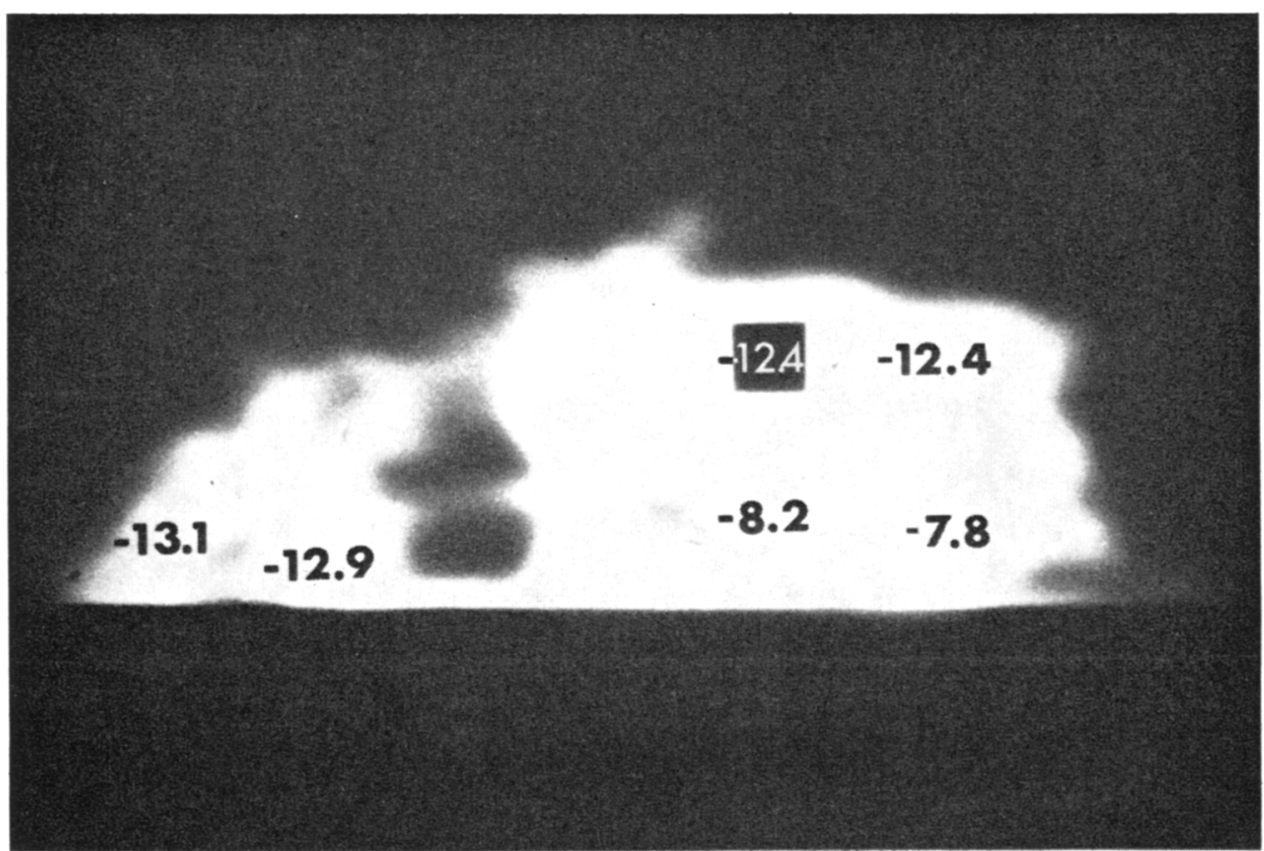

\section{March 4, 1968}

Fig. 2. Quiescent prominences showing longitudinal magnetic fields typically of only one polarity. 


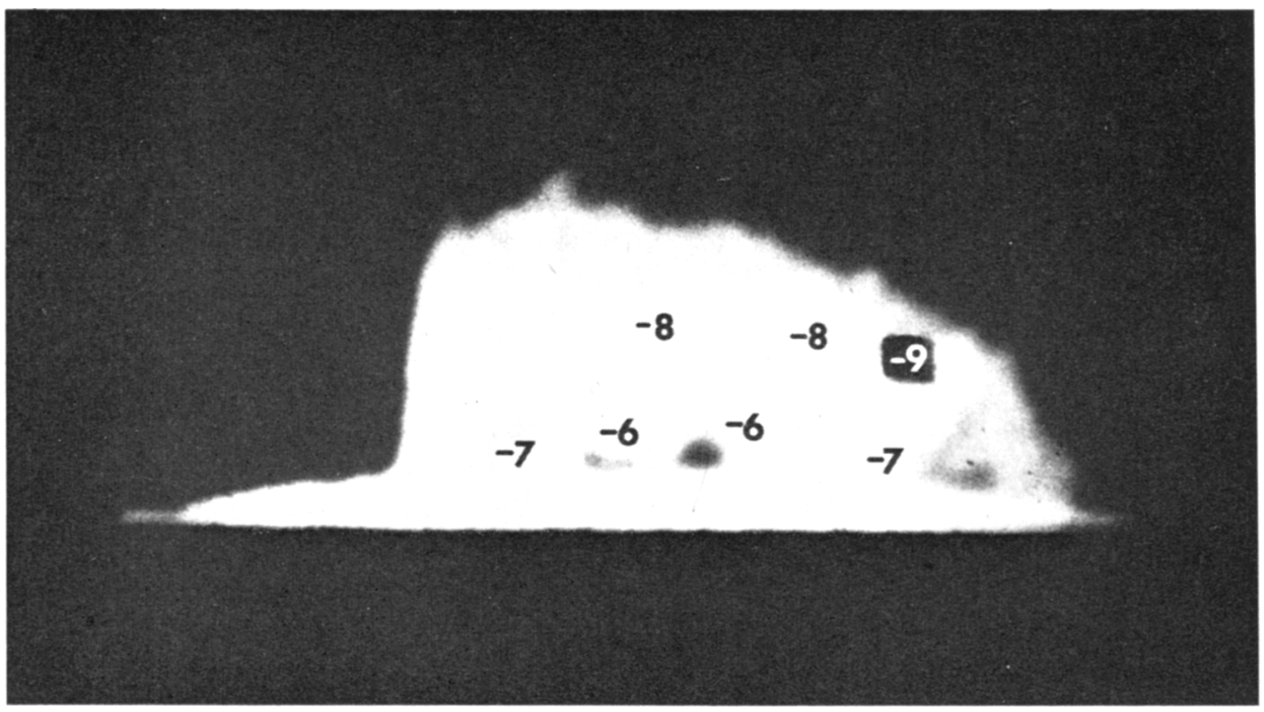

$$
\text { June } 5,1968
$$

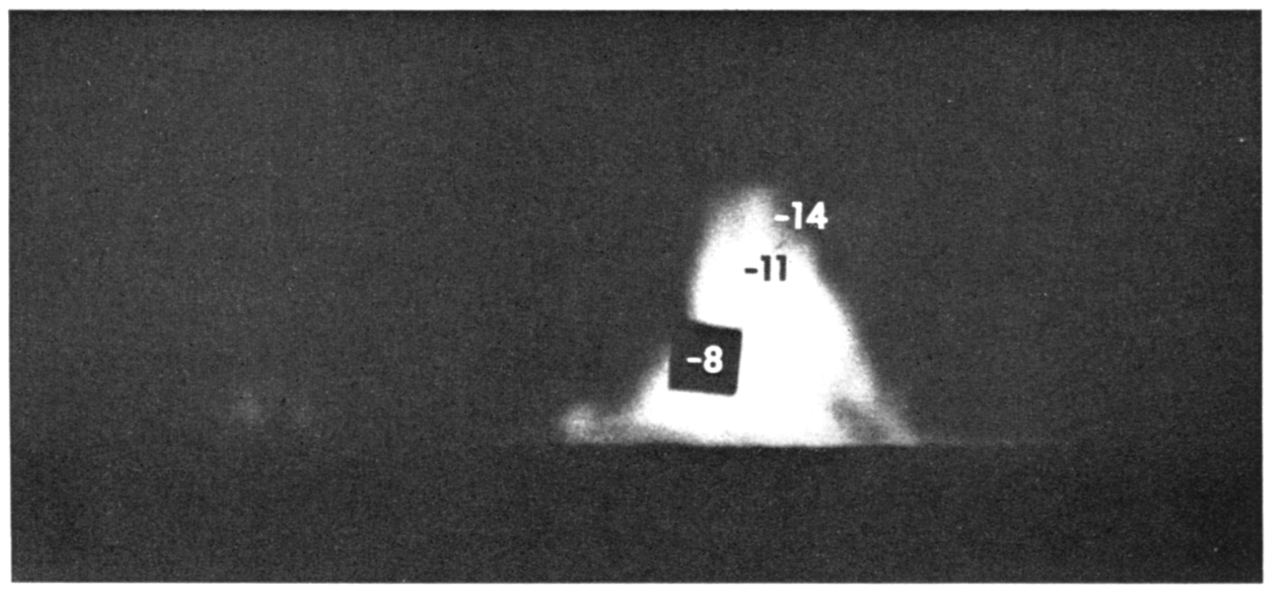

\section{March I, 1968}

Fig. 3. Quiescent prominences showing longitudinal magnetic fields increasing with height. 
TABLE I

Observed longitudinal component of magnetic field, $B_{\|}$, in quiescent prominences

\begin{tabular}{|c|c|c|c|c|c|}
\hline Date & $\begin{array}{l}\text { Position } \\
\text { angle }\end{array}$ & $\begin{array}{l}B_{\|} \\
\lambda\end{array}$ & Gauss & Gauss & Object \\
\hline June 26,1968 & 250 & $\begin{array}{l}D_{3} \\
D_{1}\end{array}$ & $\begin{array}{l}12.6 \\
10\end{array}$ & $\begin{array}{l} \pm 2 \\
\pm 4\end{array}$ & Small quiescent \\
\hline & & $\mathrm{H} \alpha$ & 9.0 & $\pm \mathbf{0 . 7}$ & Same prominence June 25 \\
\hline Sept. 8, 1968 & 325 & $\begin{array}{l}\mathrm{H} \alpha \\
\mathrm{D}_{3}\end{array}$ & $\begin{array}{l}-0.1 \\
+0.3\end{array}$ & $\begin{array}{l} \pm 0.7 \\
\pm 0.7\end{array}$ & Small quiescent \\
\hline Sept. 24, 1968 & 315 & $\begin{array}{l}\mathrm{H} \alpha \\
\mathrm{D}_{3} \\
4471\end{array}$ & $\begin{array}{l}6 \\
5.5 \\
7\end{array}$ & $\begin{array}{l} \pm 0.5 \\
\pm 0.5 \\
\pm 3\end{array}$ & Big quiescent \\
\hline Nov. 27, 1968 & 90 & $\begin{array}{l}D_{3} \\
4471 \\
D_{1} \\
D_{2} \\
b_{1}\end{array}$ & $\begin{array}{l}21 \\
21 \\
18 \\
23 \\
15\end{array}$ & $\begin{array}{l} \pm 0.5 \\
\pm 4 \\
\pm 2 \\
\pm 2 \\
\pm 4\end{array}$ & Semi-quiescent \\
\hline Jan. 16, 1969 & 59 & $\begin{array}{l}\mathrm{H} \alpha \\
\mathrm{D}_{3} \\
\mathrm{D}_{3} \\
4471 \\
\mathrm{D}_{1} \\
\mathrm{D}_{2}\end{array}$ & $\begin{array}{l}-16.6 \\
-18 \\
-15 \\
-21 \\
-20 \\
-27\end{array}$ & $\begin{array}{l} \pm 1 \\
\pm 3 \\
\pm 1 \\
\pm 3 \\
\pm 2 \\
\pm 2\end{array}$ & \\
\hline Jan. 21, 1969 & 86 & $\begin{array}{l}\mathrm{H} \alpha \\
4471 \\
\mathrm{D}_{1} \\
\mathrm{D}_{2}\end{array}$ & $\begin{array}{l}-15.7 \\
-12 \\
-15 \\
-14\end{array}$ & $\begin{array}{l} \pm 0.5 \\
\pm 6 \\
\pm 2 \\
\pm 3\end{array}$ & Part of big quiescent \\
\hline April 5, 1969 & 310 & $\mathrm{H} \alpha$ & $\begin{array}{l}-11.5 \\
\text { to } \\
-14.4 \\
-15.4\end{array}$ & +0.5 & Small quiescent \\
\hline April 24, 1969 & 297 & $\begin{array}{l}\mathrm{H} \alpha \\
4471 \\
\mathrm{D}_{3} \\
\mathrm{D}_{1} \\
\mathrm{D}_{2}\end{array}$ & $\begin{array}{l}-29.4 \\
-17 \\
-24 \\
-26 \\
-31\end{array}$ & $\begin{array}{l} \pm 4 \\
\pm 5 \\
\pm 1 \\
\pm 2 \\
\pm 2\end{array}$ & Semi-quiescent \\
\hline Aug. 16, 1969 & 130 & $\begin{array}{l}\mathrm{H} \alpha \\
\mathrm{D}_{3}\end{array}$ & $\begin{array}{l}5 \text { to } 8 \\
8 \text { to } 10\end{array}$ & $\begin{array}{l} \pm 1 \\
\pm 1\end{array}$ & Small quiescent \\
\hline Jan. 4, 1970 & 75 & $\begin{array}{l}\mathrm{D}_{3} \\
\mathrm{D}_{3} \\
\mathrm{D}_{3} \\
\mathrm{D}_{1} \\
\mathrm{D}_{1} \\
\mathrm{D}_{2} \\
\mathrm{D}_{2} \\
\mathrm{~b}_{1}\end{array}$ & $\begin{array}{l}13 \\
18 \\
14 \\
20 \\
15 \\
10 \\
13 \\
12\end{array}$ & $\begin{array}{l} \pm 1 \\
\pm 2 \\
\pm 1 \\
\pm 4 \\
\pm 4 \\
\pm 8 \\
\pm 6 \\
\pm 2\end{array}$ & Big quiescent \\
\hline Jan. 6, 1970 & 100 & $\begin{array}{l}\mathrm{H} \alpha \\
\mathrm{D}_{3}\end{array}$ & $\begin{array}{l}-29 \\
-28\end{array}$ & $\begin{array}{l} \pm 0.5 \\
\pm 1\end{array}$ & \\
\hline
\end{tabular}

\section{The Orientation of the Magnetic Field in Quiescent Prominences}

We have seen in Section 2 that the supporting magnetic field in quiescent prominences generally enters the prominence on one side, goes through the prominence plasma (with components both along and at right angles to the long axis of the prominence), 


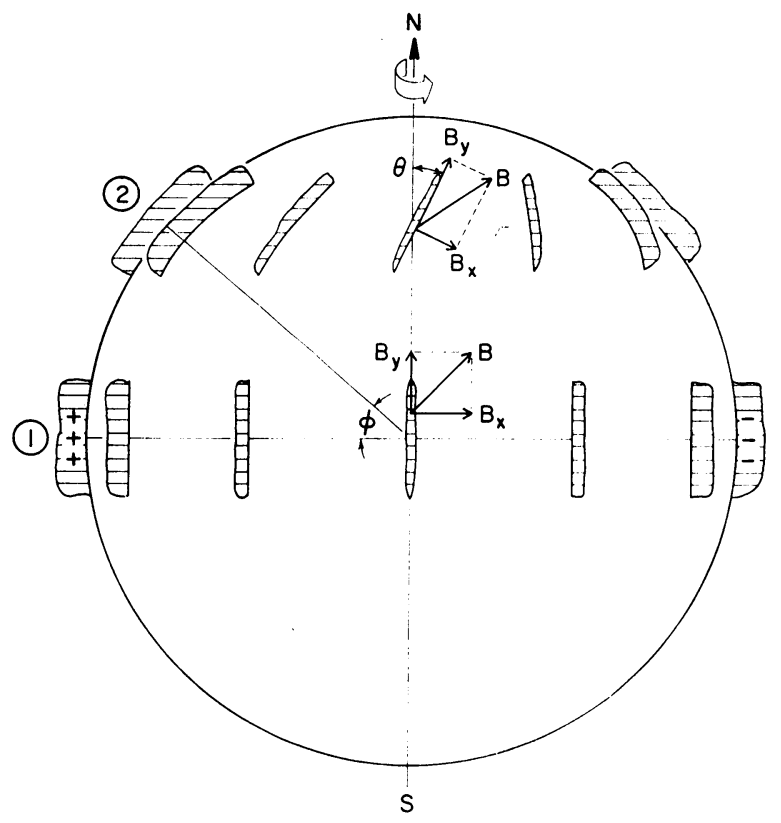

Fig. 4. Parameters defining (a) a prominence in the plane of the sky on the solar equator (Prominence 1), and (b) a prominence at latitude $\phi$ and not in the plane of the sky (Prominence 2).

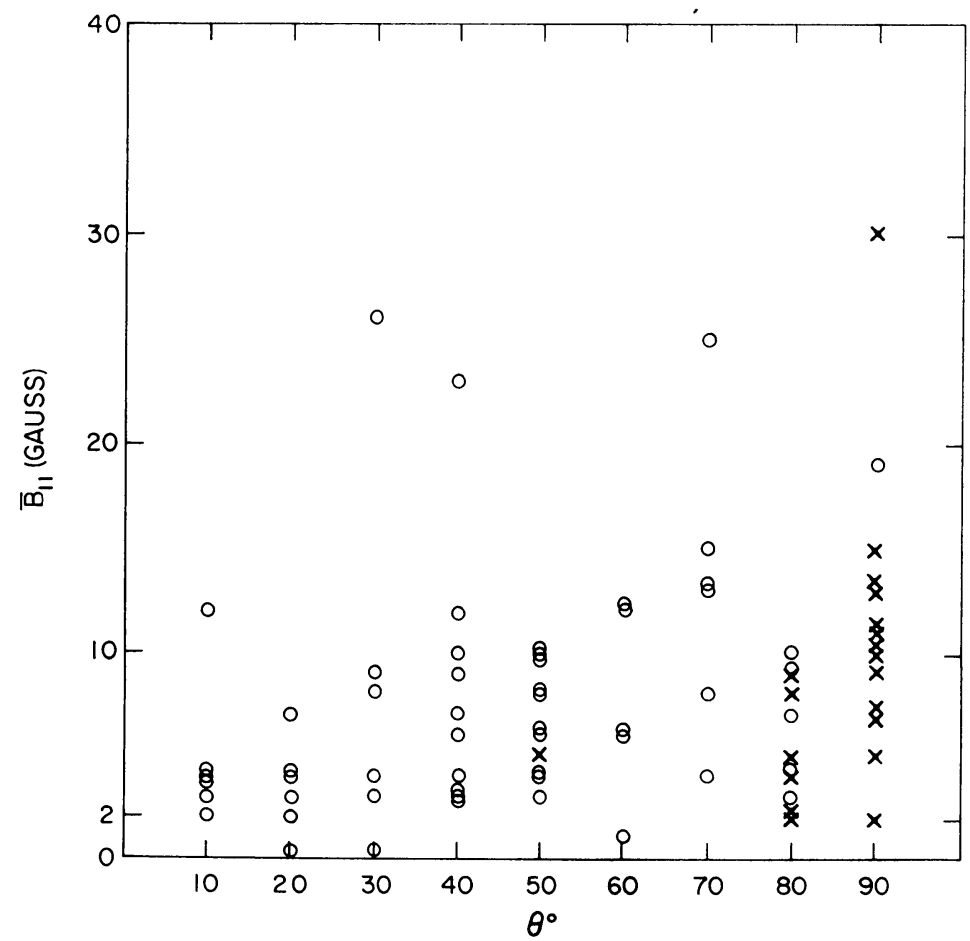

Fig. 5. The observed mean longitudinal component of the magnetic field, $\bar{B}_{\|}$, plotted against the angle $\theta$ between the north-south direction on the Sun and the long axis of the prominence (see Figure 4). 
and exits on the other side. The question arises whether we can say anything about the relative importance of the two components of the field. Let us consider an orthogonal coordinate system with $x$-axis horizontal and perpendicular to the (idealized) prominence sheet, $y$-axis horizontal and along the prominence and $z$-axis perpendicular to the photosphere. The observations on the limb of a prominence on the solar equator and in the plane of the sky give $B_{\|}=B_{x}$, see Figure 4 , prominence 1 . Often the prominence sheet does not form a plane, and even when it does, the plane may not coincide with the plane of the sky. We have studied those prominences that seem to form plane sheets and, as they move onto the disk, we have determined the angle $\theta$ that this assumed vertical plane makes with the north-south direction on the Sun, see Figure 4, prominence 2.

About $50 \%$ of the quiescent prominences observed could be approximated with the ideal cases of Figure 4. Figure 5 shows how the magnetic field $\bar{B}_{\|}$relates to the angle $\theta$. We emphasize the uncertainties involved in determining the angle $\theta$ for any prominence, but taken at face value Figure 5 indicates that the observed field $\bar{B}_{\|}$ increases with increasing angle $\theta$, i.e. $\bar{B}_{\|}$is greater the more the prominence is aligned parallel to the solar equator. This result indicates that even though the magnetic field enters a prominence on one side and exits on the other, inside the object the field tends to run along the long axis of the prominence.

\section{References}

Hanle, W.: 1924, Z. Phys. 29-30, 93.

Harvey, J.: 1959, Thesis, Univ. of Colorado.

House, L. L.: 1970a, J. Quant. Spectrosc. Radiative Transfer 10, 909.

House, L. L.: 1970b, J. Quant. Spectrosc. Radiative Transfer 10, 1171.

Hyder, C. L.: 1968, Solar Phys. 5, 29.

Lamb, F. K.: 1970, Solar Phys. 12, 186.

Lee, R. H., Harvey, J., and Tandberg-Hanssen, E.: 1969, Appl. Opt. 8, 2370.

Lee, R. H., Rust, D., and Zirin, H.: 1965, Appl. Opt. 4, 1081.

Rust, D.: 1966, Thesis, Univ. of Colorado.

Stenflo, J. O.: 1969, Solar Phys. 8, 260.

White, H. E.: 1934, Introduction to Atomic Spectra, McGraw-Hill, New York, London.

\section{Discussion}

Wiehr: How did you calibrate your magnetograph signals? I think a simple Doppler calibration is impossible since it requires a photospheric absorption line which does not exist for $\mathrm{He}_{3} \mathrm{D}_{3}$. Furthermore it would be impossible to use the calibration from an absorption line for the data from an emission line.

Tandberg-Hanssen: We calibrate it in the fairly conventional way as a Babcock magnetograph, and all the uncertainties regarding the interpretation of polarization in terms of magnetic fields are certainly with us.

Severny: (1) Do you have histograms showing the distribution of noise (modulator off), or what is the rms of noise at the brightness of a prominence?

(2) Due to thermal velocities the profiles of lines in prominences are very asymmetrical, and so a false signal can appear just due to the asymmetry of the line.

Tandberg-Hanssen: For integration times of around $10 \mathrm{~min}$ the noise in $\mathrm{H} \alpha$ is about $0.5 \mathrm{G}$.

As long as the right and left circularly polarized components have the same shape, asymmetries are eliminated. 
Jordan: In the active prominences, are the high excitation lines associated with high magnetic fields or vice-versa?

Tandberg-Hanssen: Both cases are observed. I am afraid that there is, as yet, no clearcut picture to present.

Rust: Concerning the question raised by Dr. Wiehr, I think Dr. Tandberg-Hanssen tried to answer it on a more sophisticated level than it required. The Climax magnetograph is calibrated by artifically shifting the line under observation. No reference to absorption lines on the disk is necessary.

Brueckner: Is the magnetograph arrangement built in such a way that any phase shift in the instrument will not be seen by the circular analyser, so that the known high degree of linear polarization, caused by the Hanle effect and measured in prominences cannot influence the longitudinal field measurements?

Tandberg-Hanssen: The polarization analyser is located at the prime focus, hence there is no phase shift in front of the electro-optic plate. 